\title{
Extra-embryonic endoderm cells derived from ES cells induced by GATA Factors acquire the character of XEN cells Daisuke Shimosato ${ }^{1,2}$, Makoto Shiki1,2 and Hitoshi Niwa*1,2,3
}

\begin{abstract}
Address: ${ }^{1}$ Laboratory for Pluripotent Cell Studies, RIKEN Center for Developmental Biology (CDB), 2-2-3 Minatojima-minamimachi, Chuo-ku, Kobe, Hyogo 650-0047, Japan, ${ }^{2}$ Laboratory for Development and Regenerative Medicine, Kobe University Graduate School of Medicine, 7-5-1 Kusunokicho, Chuo-ku, Kobe, Hyogo 650-0017, Japan and ${ }^{3}$ CREST (Core Research for Evolutional Science and Technology), Japan Science and Technology Agency, Honcho 4-1-8, Kawaguchi, Saitama 332-0012, Japan

Email: Daisuke Shimosato - dsk-s@cdb.riken.jp; Makoto Shiki - mshiki@cdb.riken.jp; Hitoshi Niwa* - niwa@cdb.riken.jp

* Corresponding author
\end{abstract}

Published: 3 July 2007

BMC Developmental Biology 2007, 7:80

This article is available from: http://www.biomedcentral.com/l47/-2I3X/7/80

(c) 2007 Shimosato et al; licensee BioMed Central Ltd.

This is an Open Access article distributed under the terms of the Creative Commons Attribution License (http://creativecommons.org/licenses/by/2.0), which permits unrestricted use, distribution, and reproduction in any medium, provided the original work is properly cited.
Received: 9 February 2007

Accepted: 3 July 2007

\begin{abstract}
Background: Three types of cell lines have been established from mouse blastocysts: embryonic stem (ES) cells, trophoblast stem (TS) cells, and extra-embryonic endoderm (XEN) cells, which have the potential to differentiate into their respective cognate lineages. ES cells can differentiate in vitro not only into somatic cell lineages but into extra-embryonic lineages, including trophectoderm and extra-embryonic endoderm (ExEn) as well. TS cells can be established from ES cells by the artificial repression of Oct3/4 or the upregulation of $C d x 2$ in the presence of FGF4 on feeder cells. The relationship between these embryo-derived XEN cells and ES cell-derived ExEn cell lines remains unclear, although we have previously reported that overexpression of Gata4 or Gata6 induces differentiation of mouse ES cells into extra-embryonic endoderm in vitro.

Results: A system in which GATA factors were conditionally activated revealed that the cells continue to proliferate while expressing a set of extra-embryonic endoderm markers, and, following injection into blastocysts, contribute only to the extra-embryonic endoderm lineage in vivo. Although the in vivo contribution is limited to cells of parietal endoderm lineage, Gata-induced extra-embryonic endoderm cells (gExEn) can be induced to differentiate into visceral endodermlike cells in vitro by repression of Gata6. During early passage, the propagation of gExEn cells is dependent on the expression of the Gatab transgene. These cells, however, lose this dependency following establishment of endogenous Gatab expression.

Conclusion: We show here that Gata-induced extra-embryonic endoderm cells derived from ES cells mimic the character of XEN cells. These findings indicate that Gata transcription factors are sufficient for the derivation and propagation of XEN-like extra-embryonic endoderm cells from ES cells.
\end{abstract}

\section{Background}

During early mammalian development, the zygote cleaves several times and gives rise to embryonic and extra-embryonic lineages before implantation occurs. After compac- tion in the mouse embryo, the outer cells of the morula are epithelialized and become trophectoderm (TE), while the inner cells generate the pluripotent inner cell mass (ICM). The surface of the ICM adjacent to the blastocyst 
cavity differentiates into primitive endoderm (PrE), precursor cells of the extraembryonic endoderm (ExEn) lineage. PrE subsequently differentiates into visceral endoderm (VE) and parietal endoderm (PE) [1]. VE forms layers of columnar epithelial cells covering the epiblast and contributes to the visceral yolk sac, while PE migrates along the surface of the inner TE, secreting extracellular matrix to form the Reichert's membrane and contributes to parietal yolk sac as well [2]. These ExEn lineage cells are important in embryonic development, as nutritive supports and as a determinant of the anterior-posterior axis.

In early mouse development, the GATA family zinc-finger transcription factors Gata6 and Gata4 are specifically expressed in ExEn [3]. Expression of Gata6 starts at 3.5 dpc in ICM in a salt-and-pepper pattern, which is restricted to parietal endoderm at $7.0 d p c[4,5]$. Gata6 knockout mice are embryonically lethal at $5.5-6.5 d p c$ due to defects in PrE formation and subsequent ExEn development $[4,6]$. Gata6-null embryonic stem (ES) cells fail to undergo VE differentiation in vivo and in vitro [6], and differentiation into ExEn does not occur, although Gata4-null ES cells can be induced to undergo epithelial differentiation by retinoic acid. [7]. This suggests that Gata6 function is required for early ExEn, including PrE, as well as for the development of both VE and PE.

Leukemia inhibitory factor (LIF) is required to maintain the pluripotency of mouse ES cells in conventional culture conditions; withdrawal of LIF causes ES cells to differentiate into PrE-like cells [8]. Overexpression of the POU family transcription factor Oct3/4 induced PrE-like differentiation with up-regulation of Gata4 [9], similar to the withdrawal of LIF, and overexpression of either Gata4 or Gata6 is sufficient to trigger the differentiation of ES cells into ExEn, which are similar to PE in morphology and gene expression pattern [10]. This indicates that ES cells possess the ability to differentiate into cells of the ExEn lineage, although they merely contribute to ExEn after injection into blastocysts[11].

Extra-embryonic endoderm (XEN) cells derived from blastocysts continuously propagate in vitro, while maintaining their ability to contribute to ExEn lineage cells after injection into blastocysts [12]. The morphology and expression of marker genes of XEN cells is similar to that of ES-derived PE cells induced by Gata4 or Gata6, suggesting that Gata-transfected ES cells may acquire XEN-like ExEn characteristics in vitro, although this has yet been confirmed.

Here, we report that an ExEn cell lines derived from mouse ES cells by the artificial activation of GATA factors acquire XEN-like properties. We characterized these cell lines, which we have designated gExEn cells, in compari- son with embryo-derived XEN cells. gExEn cells express specific marker genes for ExEn and differentiate into both $\mathrm{PE}$ and VE in vitro. Moreover, their contribution in vivo is restricted to the ExEn lineage, as is that of XEN cells. Although GATA activation is continuously required for the propagation of gExEn cells during early passages, these cells can propagate without artificial activation of GATA in later passages, at which time endogenous GATA factors expression is induced and maintained. We show that GATA factors play a fundamental role in establishing and maintaining gExEn cells.

\section{Results}

Continuous propagation of ExEn cells induced from ES cells by ectopic expression of Gata4 or Gata6

By functional screening of transcription factors whose expression is upregulated after induction of differentiation in ES cells, we found that the GATA-family transcription factors Gata4 and Gata6 could induce differentiation toward the ExEn lineage [10]. Upon ectopic expression of Gata4 or Gata6, ES cells differentiated into dispersed refractive cells that resembled PE cells and expressed PE marker genes such as Sparc (secreted acidic cysteine rich glycoprotein) and Plat (tPA; plasminogen activator, tissue), indicating that activation of Gata4 and Gata6 is sufficient for inducing PE-like ExEn differentiation in ES cells.

XEN cells derived from blastocysts were recently reported to show very similar morphology to Gata4 or Gata6 induced PE cells derived from ES cells [12]. XEN cells were robust on mouse embryonic fibroblast (MEF) feeder layer or $70 \%$ conditioned medium (CM) from MEF for several passages. However, the ability of ExEn cells derived from ES cells to propagate following ectopic expression of Gata4 or Gata6 had not been determined. We therefore assessed the ability of Gata6 and Gata4 episomal transfectants with PE-like morphology to propagate in prolonged culture. We found, however, that these cells could be passaged fewer than three times in the culture conditions used for XEN cells (Table 1). Since the episomal expression system tends to become destabilized after induction of differentiation (Niwa, H., unpublished), we tested SKG612 [10] and EBRTc-G6 [13] ES cells, both of which carry integrated copies of tetracycline (Tc)-inducible Gata6 transgenes and differentiate into PE-like cells after induction of ectopic Gata6 expression following withdrawal of Tc (Fig. 1A or 1C). We found that, although SKG612-derived ExEn cells (Fig. 1B) could be passaged fewer than three times (Table 1), EBRTc-G6-derived ExEn cells (Fig. 1C) propagated continuously for more than 10 passages on MEF (Table 1), suggesting that these PE-like cells acquire XEN cell-like ability of proliferation.

To further investigate the role of the GATA factors on ExEn differentiation and their XEN cell-like characteristics, we 
Table I: Culture conditions of three PE-like cells

\begin{tabular}{|c|c|c|c|c|}
\hline \multirow{2}{*}{$\begin{array}{c}\text { coating } \\
\text { exo.Gata6 }\end{array}$} & \multicolumn{2}{|c|}{ MEF } & \multicolumn{2}{|c|}{ Gelatin } \\
\hline & ON & OFF & ON & OFF \\
\hline MGZ5 & \pm & - & - & - \\
\hline SKG6I2 & + & - & \pm & - \\
\hline EBRTc-G6 & +++ & \pm & \pm & \pm \\
\hline 5G6GR & +++ & + & +++ & \pm \\
\hline
\end{tabular}

PE-like cells were derived from 5G6GR, EBRTc-G6 and SKG6I2 cells and grown with or without MEF, and in the presence or absence of Dex (5G6GR) or Tc (EBRTc-G6 and SKG6I2). Passage numbers: +++ , more than I0; +, 3-I0; \pm , less than 3; - impossible to grow.

established another inducible activation system for the GATA factors in ES cells by introducing a chimeric transgene composed of full-length Gata4 or Gata6 and the human glucocorticoid receptor ligand-binding domain (G4GR and G6GR, respectively). Introduction of $p C A G-$ G4GR-IP or -G6GR-IP into EB5 ES cells resulted in the establishment of the ES cell lines, 5G6GR (Fig. 1G) and 5G4GR (data not shown), respectively. GFP-tagged G6GR showed that, in the absence of dexamethasone (Dex), the chimeric transgene products were kept inactive in the cytoplasm (Fig. 1E), whereas, in the presence of Dex, they translocated into the nucleus (Fig. 1F), indicating that these chimeric molecules were properly regulated. Although parental EB5 ES cells had no morphological changes by the administration of Dex (data not shown), treatment with Dex altered the morphology of these 5G6GR or 5G4GR ES cells into dispersed, refractive and satellite type, reminiscent of PE cells. These cells, designated g6ExEn (Fig. 1I) and g4ExEn (data not shown) as found in episomal transfectants of Gata4 and Gata6, respectively, and were not similar to PrE induced by the withdrawal of LIF (Fig. 1H). Thus, these results indicated that the hormone-inducible GATA factors mimic the function of native GATA factors in ES cells.

g4ExEn and g6ExEn cells each had two distinct morphologies, depending on the culture conditions on gelatinized dishes. Dispersed refractive cells were observed in Gata6 or Gata4 transfectants under low-density culture conditions (Fig. 1I), whereas an epithelial sheet-type morphology was observed under high-density conditions (Fig. 1I, squares). In the presence of Dex, these cells could be expanded on MEF for more than 10 passages (Fig. 1J), similar to results for EBRTc-G6-derived ExEn cells (Table 1 ). These data suggested that gExEn cells acquire an ability to proliferate similar to that of XEN cells.

\section{High level of constitutive activation of GATA factors can substitute for the MEF requirement}

Withdrawal of MEF was found to induce differentiation of XEN cells by reduction of Gata4 or induction of Afp
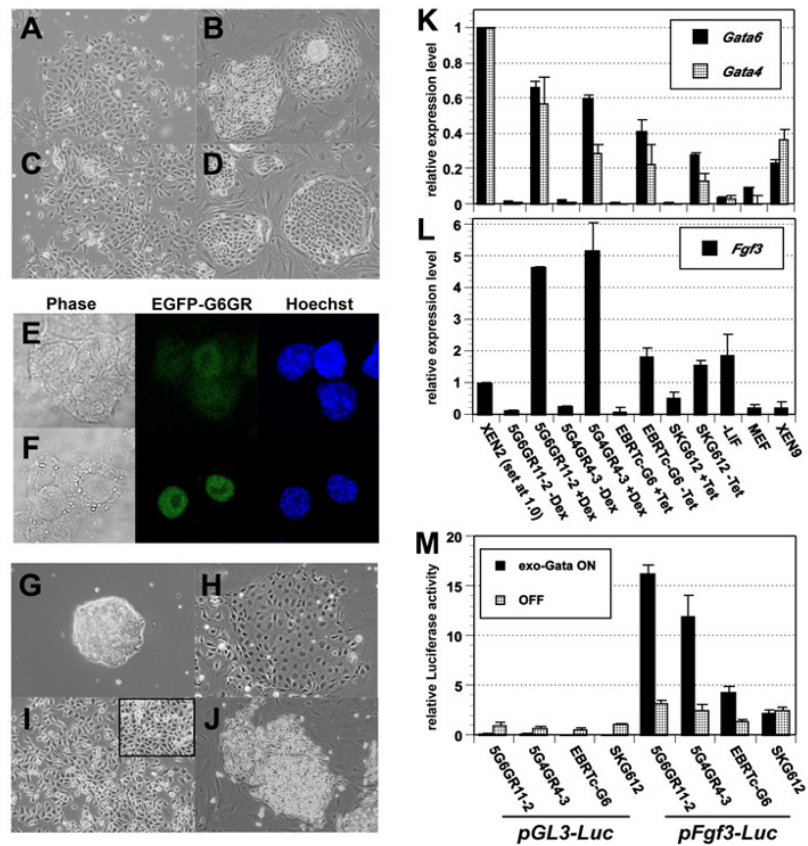

Figure I

Induction of gExEn cells from ES cells by inducible Gata6 or Gata4 expression systems. (A-D) Morphology of differentiated SKG6I2 (A, B) and EBRTc-G6 (C, D) cells induced by withdrawal of Tc on gelatinized dishes $(A, C)$ or under MEF culture conditions for 5 days $(B, D)$. (E, F) Confocal microscopic image of ES cells with introduced pCAG-EGFP-G6GR $2 \mathrm{hr}$ after the addition of $70 \% \mathrm{EtOH}$, without (E) and with (F) Dex. The left panels show phase-contrast images, the center panels show localization of EGFP-G6GR monitored by EGFP fluorescence, and the right panels show nuclei stained with Hoechst33342. (GJ) Morphology of 5G6GR I I-2 ES cells with or without Dex.

5G6GRII-2 ES cells were grown in the presence (G) or absence $(\mathrm{H})$ of LIF for 5 days, or were treated with $100 \mathrm{nM}$ Dex for 5 days on gelatinized dishes (I, high-density conditions in inset) or on MEF (J). (K) Expression levels of endogenous Gatab and Gata4 in ES-derived ExEn cells 4 days after activation of exogenous Gata on gelatinized dish cultures or PrE induced by withdrawal of LIF for 5 days. XEN2 and XEN9 cells; derived from embryo and MEF-dependent propagation. MEF; MEF alone. All values were normalized relative to the level of Gapdh and plotted relative to levels of expression in XEN2 cells. (L) Expression levels of endogenous Fg 3 in ES-derived ExEn cells 4 days after activation of exogenous GATA on gelatinized dish cultures, or PrE induced by withdrawal of LIF for 5 days. XEN2 and XEN9 cells; derived form embryo and MEF-dependent propagation. MEF; MEF alone. All values were normalized relative to the level of Gapdh and plotted relative to levels of expression in XEN2 cells. (M) GATA-dependent enhancer activity of the element containing GATA-binding site of pFg/3-luc in ES-derived ExEn cells. Reporter plasmids were transfected into 5G6GRII-2 and 5G4GR4-3 ES cells followed by culture with (exo-GATA ON) or without Dex (OFF), or EBRTC-G6 and SKG6 I 2 ES cells followed by culture with (OFF), or without Tc (exo-GATA ON). Relative expression levels of pFgf3-luc (filled; exo-GATA ON, hatched; OFF) are shown. All results were normalized relative to the luciferase activities of $p C M V-R L$ and plotted relative to the luciferase activities of $p G$ L3-luc in Dex-non-treated 5G6GRII-2, set at I.0. 
(alpha-fetoprotein), a marker for VE in early embryos [12]. Both SKG612 and EBRTc-G6-derived ExEn cells showed limited capacity to propagate on gelatin-coated dishes in the absence of Tc, indicating an absolute requirement for MEF, as observed in XEN cells (Table 1 and Fig. 1A and 1C). In contrast, gExEn cells, derived from G4GR or G6GR ES cells induced by treatment with Dex, could be serially passaged on gelatinized dishes at about 1:40 dilution every 3 or 4 days (Fig. 1I). The culture period reached at least 40 passages for 4 g6ExEn lines (2-2, 2-3, 11-2, 11-3), and at least 10 passages for 2 g4ExEn lines $(1-1,4-3)$ on gelatinized dishes with no apparent senescence or reduction in viability (data not shown), indicating that they had lost their requirement for MEF for continuous propagation.

What is the molecular basis of the requirement of MEF for XEN cells? According to the original report by Kunath et al., MEF feeder layers can be substituted by the MEF-CM, indicating that one or more soluble factors secreted by MEF is required for XEN cells. If this signal is required for the transcriptional activation of Gata6 and/or Gata4, a constitutive supply of GATA factors from the transgenes beyond the threshold level may override the MEF dependency. Since we applied different chimeric transgenes in different inducible systems for activation of GATA factors and it was suggested that Gata6 and Gata4 possess crossand auto-activation systems, simple measurement of the amount of transcripts for these transgenes, as well as the endogenous gene, was not a suitable indicator of the net GATA activity achieved in these transgenic ES cells. To achieve this, we used two different approaches; (1) transcriptional quantification of the endogenous GATA target genes, and (2) measurement of the activity of GATAdependent reporters. When the luciferase (luc) reporter carrying the Gata6 promoter and the first intron ( $p$ Gata6-luc), where the auto-regulatory elements are found in other Gata family genes [14], was introduced in 5G6GR and 5G4GR ES cells, it was significantly activated within 24 hours in the presence of Dex in both cell lines, strongly suggesting the direct activation of this reporter by GATA6 and GATA4 ' [see additional file 1]'. We quantified endogenous Gata6 in ES cells carrying various types of inducible Gata6 transgenes and found that the expression levels of Gata6 in G6GR and G4GR cells with Dex were high, that of EBRTc-G6 with Tc was moderate, and that of SKG6 with Tc was low, but still significantly higher than that of PrE cells induced by withdrawal of LIF (Fig. 1K). The expression levels of Gata6 were varied in two XEN cell lines established from blastocysts (XEN2 and XEN9), but the ranges of the expression levels were comparable to those of ES-derived ExEn cells (Fig. 1K). Similar results were obtained for Gata4 expression, suggesting the presence of its auto-and cross-regulation by GATA factors. As the additional indicator, we chose Fibroblast growth factor (Fgf)-3 because the direct regulation of its promoter by GATA4 has been reported [15]. We confirmed that the activation of the $p F g f 3$-luc and $p F g f 3-t k$-luc reporter was comparable to that of $p$ Gata6-luc at 24 hours after induction of exogenous GATA activities (Fig. 1M and ' [see additional file 1]') and found that either the activities of $p F g f 3-l u c / p F g f 3-t k-$ $l u c$ or the transcription levels of endogenous $F g f 3$ at 4 days after induction of exogenous GATA activities were consistent with the hierarchy of the Gata6 expression levels in these ExEn cells (Fig. 1L and $1 \mathrm{M}$ ), indicating that the induced GATA activity is highest in 5G6GR and 5G4GR, moderate in EBRTc-G6 and lowest in SKG612. This indicates a tight relationship between the ability to self-renew in the absence of MEF and the induced activity of constitutive GATA factors independent to exogenous signal, suggesting a role for the maintenance of GATA activity at a high level in the propagation of XEN cells under feederfree conditions.

\section{Expression of ExEn marker genes in gExEn cells}

To investigate the detailed characteristics of g4ExEn and g6ExEn cells, we analyzed marker gene expression in passage 10 cells by quantitative PCR (Q-PCR). We chose Gata4 [16], Gata6 [4], Sox7 and Sox17 [17], and Disabled homolog 2 (Dab2; [18]) as ExEn markers, which are markers for ExEn, and found that all were induced in gExEn cells at much higher levels than in PrE from ES cells induced by the withdrawal of LIF (Fig. 2A). The expression levels of these markers were about 2 -fold higher in g6ExEn than in g4ExEn cells, which might reflect functional differences between Gata6 and Gata4 as previously reported, in which GATA6 is an upstream regulator of Gata4 while GATA4 is a negative regulator of Gata6 [6,7].

The level of expression of the PE markers Plat [19], Snail [20], and Sparc [21] in g6ExEn and g4ExEn cells was similar to that in PrE cells (Fig. 2B), whereas expression of Follistatin (Fst; [22]) and Parathyroid hormone receptor 1 (Pthr1; [23]) was much higher in g6ExEn and g4ExEn cells than in PrE cells (Fig. 2C).

The VE markers Afp [24], Foxa2 (Hnf3b; [25]), Indian hedgehog (Ihh; [26]), Transthyretin (Ttr; [27]), Retinol-binding protein (Rbp; [28]), Apolipoprotein E (ApoE; [29]), and Cbp/p300-interacting transactivator with Glu/Asp-rich carboxy-terminal domain 1 (Cited1/Msg1; [30]) were also induced to an equivalent or higher extent in g6ExEn and g4ExEn cells than in PrE cells (Fig. 2D). In contrast, the pluripotent cell markers Oct3/4 (Pou5f1), Nanog and Zfp42/Rex1, as well as the trophectoderm markers $C d x 2$, Hand 1 and Psx 1 were hardly detected in gExEn cells (Fig. $2 \mathrm{E}$ and data not shown). These data fit the marker gene expression profile of XEN cells reported previously. 

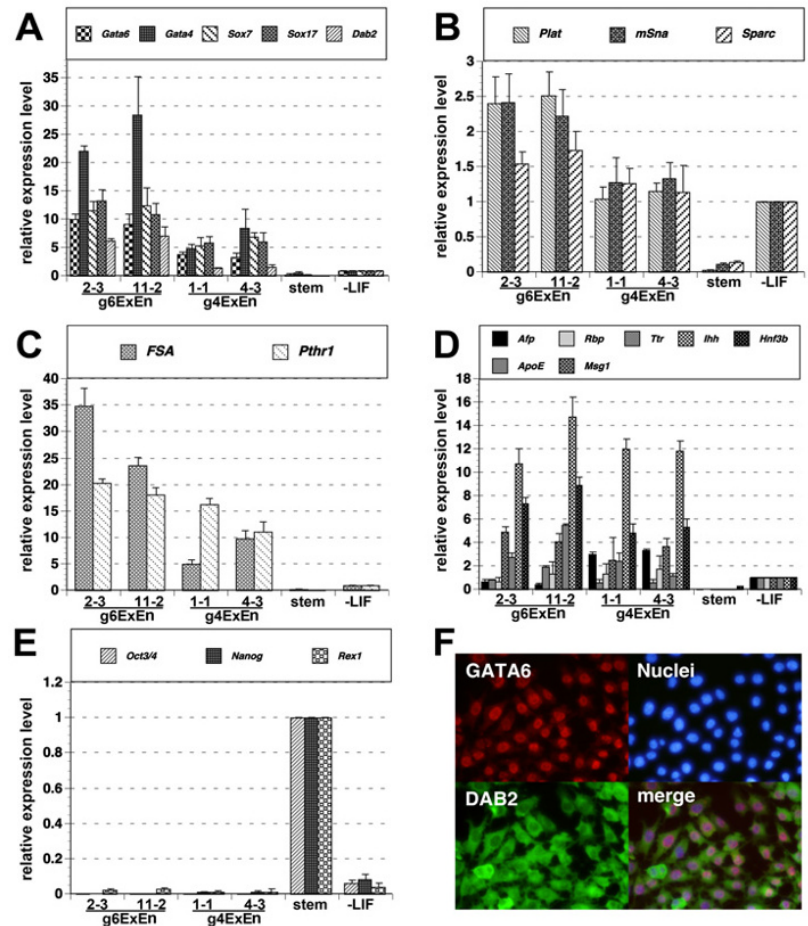

\section{$\mathbf{F}$}

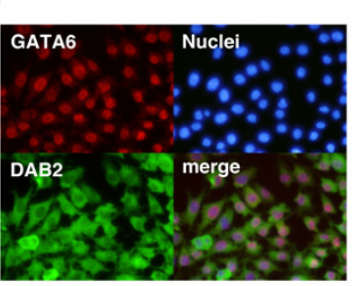

\section{Figure 2}

Marker gene expression of gExEn cells. (A-E) Q-PCR analysis of gene expression in Dex-treated gExEn cells after 10 passages. ExEn markers (A), PE markers (B and C), VE markers (D) and stem cell markers (E). Relative expression levels of the indicated marker genes in two independent clones of g6ExEn (2-3 and I I-2) and g4ExEn ( $\mathrm{I}-\mathrm{I}$ and 4-3) and in 5G6GR ES cells, in the presence and absence of LIF for 5 days, are shown. All results were normalized relative to the level of expression Gapdh and plotted relative to expression levels in 5G6GR-derived PrE without LIF (A-D) or that in 5G6GR ES cells (E). (F) g4ExEn4-3 were stained with antiGATA6 (red), anti-DAB2 (green), and nuclear staining by Hoechst33342 (blue). The lower right panel shows marginal images.

Immunohistochemical analysis showed that virtually all g4ExEn4-3 express GATA6 in the nucleus (Fig. 2F, red) and DAB2 in the cytoplasm (Fig. 2F; green), similar to embryo-derived XEN cells (data not shown), indicating that gene expression profile of gExEn cells is homogeneous, as judged by their morphology.

\section{g4ExEn and g6ExEn cells contribute to the parietal endoderm lineage in vivo}

To determine the in vivo differentiation potential of gExEn cells, we performed chimera analysis with cell lines carrying $p C A G-E G F P-I Z, 5 G 4 G R-G F P$ and 5G6GR-GFP. These
Table 2: Blastocyst injection data

\begin{tabular}{cccccc}
\hline line name & $\begin{array}{c}\text { Number } \\
\text { of } \\
\text { deciduas }\end{array}$ & $\begin{array}{c}\text { Number } \\
\text { of } \\
\text { embryos }\end{array}$ & \multicolumn{2}{c}{ Number of embryos } \\
embryonic & ExEn \\
\hline ES & (G6GR-Dex) & 36 & $25(70 \%)$ & $16(44 \%)$ & 0 \\
g6ExEn & $2-2$ & 6 & 6 & 0 & 2 \\
& $2-3$ & 25 & 10 & 0 & 8 \\
& $11-2$ & 79 & 35 & 0 & 14 \\
& $11-4$ & 15 & 4 & 0 & 1 \\
& & & & & \\
\hline \multirow{6}{*}{ g4ExEn } & total & 125 & $54(43 \%)$ & 0 & $25(20 \%)$ \\
& $1-1$ & 13 & 8 & 0 & 4 \\
& $4-3$ & 18 & 9 & 0 & 6 \\
& $4-5$ & 13 & 5 & 0 & 2 \\
\hline & total & 54 & $22(41 \%)$ & 0 & $12(22 \%)$ \\
\hline
\end{tabular}

4 g6ExEn cell clones (2-2, 2-3, II-2, I I-4) and 3 g4ExEn cell clones (I$1,4-3,4-5)$ were used to generate chimeras by blastocyst injection.

cell lines differentiated into gExEn and showed strong EGFP expression, with or without Dex treatment, which was sufficient for the detection of their progeny cells at the single-cell level in chimeric embryos.

When 5G6GR-GFP ES cells, without Dex treatment, were injected into wild-type $\mathrm{C} 57 \mathrm{Bl6} / 6 \mathrm{~J}$ blastocysts and transferred into the uteri of pseudopregnant mice, they generated 16 chimeric embryos, in which the EGFP-positive cells contributed only to the embryonic portion and were never found in the extra-embryonic lineage (Fig. 3A, B and Table 2). In contrast, when g4ExEn-GFP and g6ExEn-GFP cells derived from the Dex-treated ES cell lines 5G6GFPGR and 5G4GFP-GR, respectively, were injected after several passages, they contributed exclusively to the extraembryonic yolk sac, with a scattered pattern, a feature of $\mathrm{PE}$ in vivo, in the chimeric embryos (Fig. 3C-F and Table 2): This result was previously observed for embryoderived XEN cells [12]. However, in all chimeras, the contribution of the gExEn cells was restricted to the parietal yolk sac. A similar tendency was found for XEN cell chimeras, in which XEN cells in VE were observed in only 1 in 50 chimeras [12]. These findings indicate that g4ExEn and g6ExEn cells derived from ES cells by artificial activation of Gata4 or Gata6 have the potential to contribute to only PE in vivo.

\section{Activation of exogenous Gata factors is required for propagation of gExEn cells in the early passage period} Although in the presence of Dex g4ExEn and g6ExEn cells are robust, even in the absence of MEF (Fig. 4A, C), these cells gradually ceased to propagate and their morphology became flatter after withdrawal of Dex (Fig. 4B, D). Similar changes were observed in gExEn cells cultured on MEF, 


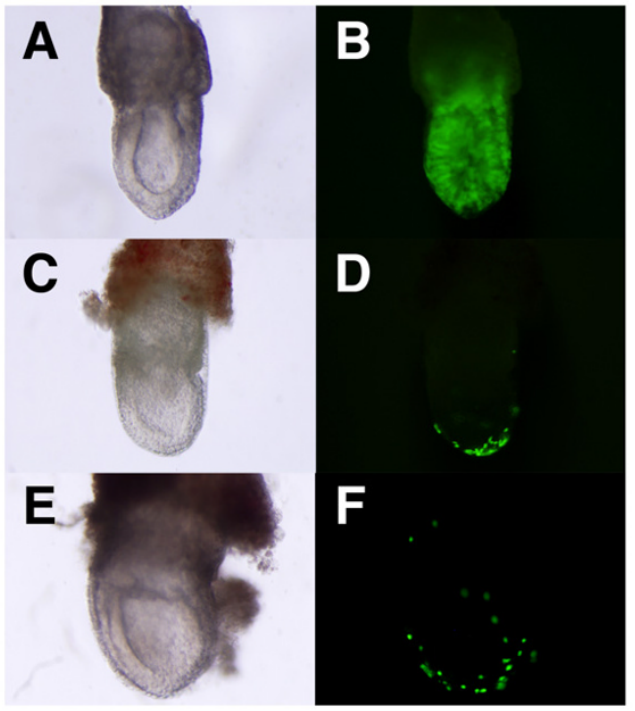

Figure 3

Contribution of gExEn cells to ExEn lineage in vivo. (A, B) $8.5 \mathrm{dpc}$ chimeric embryos derived from 5G6GR-GFP ES cells. 5G6GR-GFP ES cells, kept in undifferentiated state without Dex, give rise to embryonic chimeras. The $8.5 \mathrm{dpc}$ chimeric embryos with g6ExEn-GFP (C, D) derived from 5G6GR-GFP ES cells or g4ExEn-GFP cells (E, F) derived from 5G4GR-GFP ES cells, cultured in the presence of Dex for the activation of GATA-GR after several passages, contributed only to the distal parietal yolk sac.

suggesting that continuous activation of Gata-GR is absolutely required for the propagation of gExEn cells.

To confirm the status of gExEn cells with or without exogenous GATA activity, we assayed expression of several VE and PE marker genes by Q-PCR. Expression of endogenous Gata4 and Gata6 was reduced by inactivation of GATA6-GR in g6ExEn cells following withdrawal of Dex (Fig. 4E). In addition, expression of the ExEn marker genes Sox7 and Sox17, and the PE marker genes Plat, Snail and Pthr1 were decreased after withdrawal of Dex, whereas the VE marker genes Afp Hnf3b, Ihh, Ttr, Rbp, ApoE and Cited 1 were increased in parallel (Fig. 4E, F). These findings are similar to the gene expression profile in differentiated XEN cells induced by the withdrawal of MEF, in which decreasing expression of Gata4, and Gata6 and several PE marker genes, and increasing expression of VE markers including $A f p$, was observed [12]. Therefore, withdrawal of exogenous GATA 6 activity induces differentiation of gExEn cells, indicating that maintenance of GATA6 activity is required for propagation of gExEn cells.
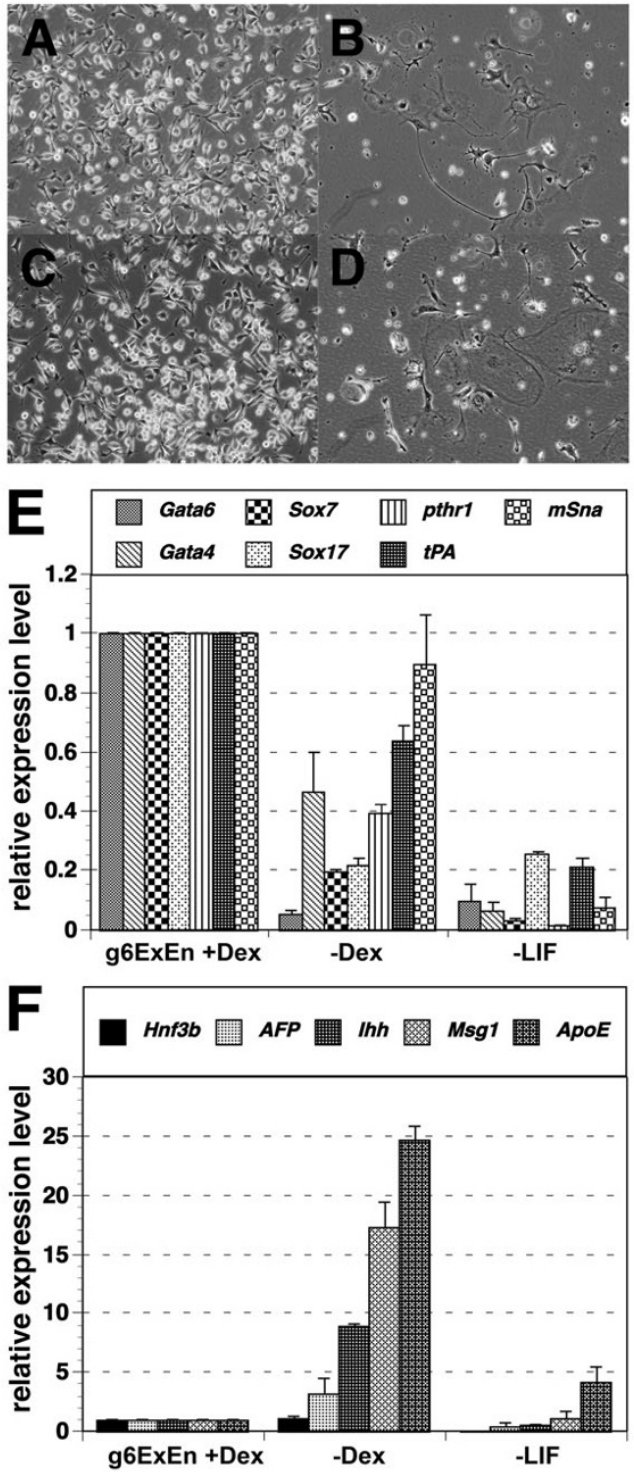

\section{Figure 4}

Effect of the extinction of exogenous GATA activity in gExEn cells. (A-D) Photomicrographs of g6ExEn I I-2 (A, $B)$ and g4ExEn4-3 (C, D) cells cultured with (A or C) or without ( $B$ or $D)$ Dex for 4 days after 2 passages in the presence of Dex. (E, F) Q-PCR analysis of PE or VE marker gene expression in g6ExEn I I-2 cells, with or without Dex. Withdrawal of Dex after 2 passages in the presence of Dex decreased expression of a set of PE marker genes (E), while expression of VE marker genes increased in parallel (F). All results were normalized relative to expression of Gapdh and plotted relative to the expression level in Dex-treated g6ExEn I I-2 cells. 


\section{Establishment of endogenous Gata expression restores dependency on exogenous Gata activity}

Interestingly, over about 5 passages, the dependency of gExEn cells on exogenous Gata activity was gradually lost and they became able to propagate without Dex. Analyses of marker gene expression in these late passage gExEn cells revealed that the expression levels of endogenous Gata4 and Gata6 were slightly higher than in early passage gExEn cells (Fig. 5A). In contrast to early passage gExEn cells (Fig. $4 \mathrm{E}$ ), the expression levels of endogenous Gata4 and Gata6 were maintained after removal of GATAF6-GR activity by withdrawal of Dex (Fig. 5B). These data indicated that the positive auto-regulatory loop that maintains expression of endogenous Gata factors was gradually established during cell culture.

To further determine whether Gata6 activity is required to maintain propagation of late passage gExEn cells, we performed a loss-of-function assay by silencing Gata6 expression using a short-hairpin RNA-mediated knockdown strategy. pSil-H1puro expresses short hairpin RNA under the control of the mouse H1-RNA gene promoter. A vector targeting Gata6, pSil-shG6, was transfected into Dex-independent puromycin sensitive g6ExEn cell line, 1D3, and the transfectants were cultured for $48 \mathrm{hr}$ under puromycin selection and then analyzed for ExEn marker expression by quantitative PCR. Transfection efficiency in 1D3 cells was monitored by transient expression of EGFP using pCAG-EGFP-IP vector transfected by the same protocol (Fig. 6A, B). FACS analysis showed that about 95\% of the cells were EGFP-positive (Fig. 6C).

The level of expression of endogenous Gata6 in the 1D3 cells transfected with pSil-shG6 was about $50 \%$ of that in control cells transfected with pSil-H1puro (Fig. 6F). After puromycin selection, the control cells exhibited no morphological changes (Fig 6E), whereas the 1D3 cells stopped propagating and showed altered morphology, similar to that observed during VE-like differentiation induced by withdrawal of Dex during the early passage period (Fig. 6D), including a 3-fold upregulation of $A f p$ relative to control cells (Fig. 6F). These data suggested that Gata6 is absolutely essential for the propagation of gExEn cells.

\section{Discussion}

The systematic in vitro differentiation of ES cells represents a powerful tool for analyzing the molecular mechanisms controlling pre-implantation development [31]. However, careful comparison of events observed in vitro and in vivo is required to use this model system properly. We have characterized gExEn cells generated in vitro from ES cells by the artificial activation of GATA factors and confirmed that they mimic the characteristics of XEN cells. gExEn cells can be propagated continuously on gelati-
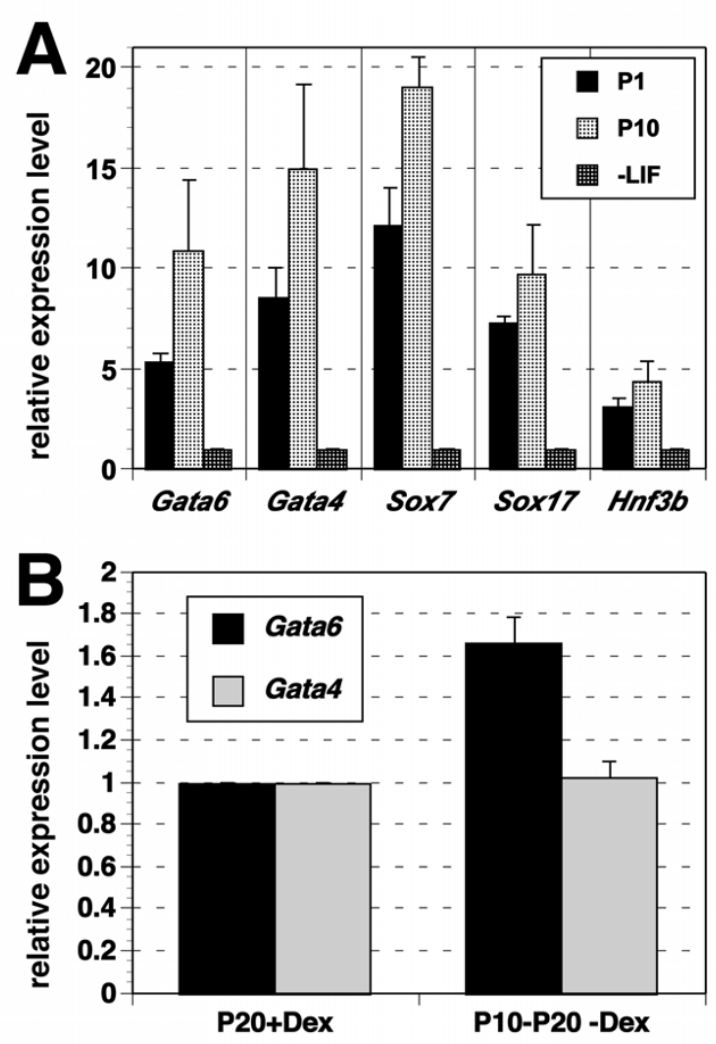

Figure 5

Expression of endogenous Gata4 and Gata6 in latepassage gExEn cells. (A) Marker gene expression in late passage: g6ExEn cells expressed endogenous Gata4 and Gata6 slightly higher than in early passage g6ExEn cells. All results were normalized relative to expression of Gapdh and plotted relative to the expression level in 5G6GR-derived PrE without LIF for 5 days. (B) After removal of Gata6-GR activity by withdrawal of Dex from passage 10 to passage 20 (PI0-P20), the expression levels of endogenous Gata4 and Gata6 were maintained as same amount level in the presence of Dex culture condition (P20). All results were normalized relative to expression of Gapdh and plotted relative to the expression level in P20 g6ExEn cells.

nized dishes by constitutive activation of exogenous GATA activity, independent of the MEF-derived signal, and contribute to ExEn in chimeric embryos, as do XEN cells. Although the in vivo contribution of gExEn cells is limited to PE, these cells differentiate in vitro into cells morphologically and genetically similar to VE cells. In addition, we confirmed that Gata6 is important for the propagation of gExEn cells. These data clearly indicate that ectopic and continuous activation of GATA4 or GATA6 is sufficient to trigger proper differentiation of ES cells into the ExEn lineage. 

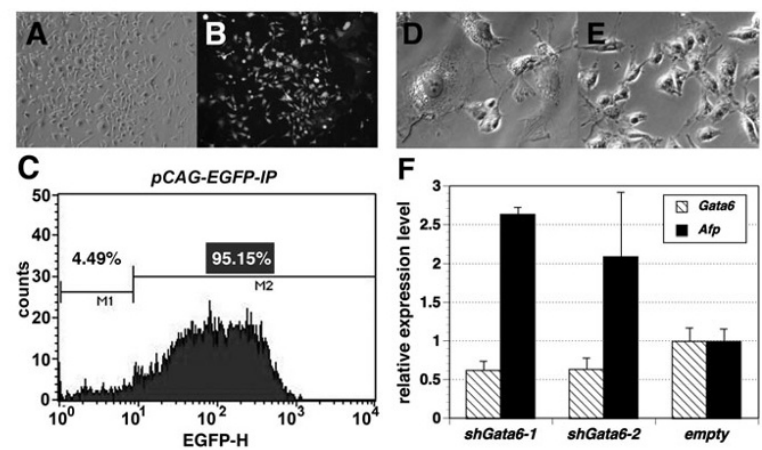

\begin{abstract}
Figure 6
Knock-down of Gata6 in late-passage gExEn cells. (AC) Efficient transfection of g6ExEn cells by EGFP expression vector. After drug selection, almost all transfectants showed GFP expression microscopically (A: phase-contrast image, B: fluorescent image for EGFP), which was confirmed by FACS analysis for EGFP fluorescence (C). (D, E) Morphology of g6ExEn cell line, ID3, transfected with the Gatab silencing vector pSil-G6 (D) or empty vector (D) after 5 days transfection. (F) Expression level of Gatab and Afp in Gata6-silenced ID3 cells by Q-PCR at day 5 . Results were normalized relative to expression of Gapdh and plotted relative to expression level in ID3 cells transfected with pSil-HI puro.
\end{abstract}

Lineage specification by tissue-specific transcription factors is a key step in development. In mouse blastocysts, there are three cell lineages, ICM, TE and ExEn, with various cell lines derived from each. To date, several ExEn cell lines have been described, including the rat yolk sac carcinoma line L2 [32], the RE1 line from a rat blastocyst [33] parietal endoderm cells (PEC; [34]) and XEN cells [12] from mouse blastocysts. Of these, XEN cells are regarded as the best model of ExEn development in vitro because of their origin and characteristics in vivo after injection into blastocysts. However, the molecular mechanisms of derivation and propagation of XEN cells have not yet been analyzed, although the functions of GATA factors in XEN cells were suggested by both gain- and loss-of-function studies in vitro and in vivo, showing that Gata4 and Gata6 were necessary and sufficient to commit cells to the ExEn lineage $[4,6,10]$.

We have clearly shown here that GATA factors play a central role in the induction and maintenance of gExEn cells. Although transient induction of ectopic GATA4 or GATA6 activity is sufficient to induce differentiation of ES into gExEn cells (data not shown), inactivation of the exogenous GATA activity in the early passage period prevented their propagation and the induction of terminal differentiation. In the late passage period, gExEn cells were freed from their dependency on exogenous GATA activity, but still had a tight requirement for endogenous GATA expres- sion. These findings indicate that gExEn cell propagation is dependent on GATA factors, and that this may also be applicable to embryo-derived XEN cells.

Although XEN cells derived from blastocysts grow robustly on MEF or in medium supplemented with $70 \%$ MEF-CM, which contains many unknown factors, we found that gExEn cell propagation is dependent only on the high level of the induced activation of GATA4 or GATA6, without any exogenous factors. In contrast, EBRTc-G6-derived ExEn cells, which showed weaker expression of endogenous Gata6 following induction of exogenous Gata6 than gExEn cells, mimic the MEFdependency of XEN cells. Since the requirement for MEF can be satisfied by high-levels of GATA factors, the soluble factors contained in MEF-CM may activate the expression of endogenous GATA factors, as we hypothesized. To date, we have tested the activity of several candidate soluble factors to substitute the role of MEF feeders, but neither FGF3 [15], a soluble factor abundantly expressed in PE, nor parathyroid hormone-like peptide (Pthih/PTHrP; [23]) secreted from $\mathrm{TE}$, the ligand of the $\mathrm{PTHrP}$ receptor expressed in PE, can substitute for activation of GATA-GR fusion protein by Dex to maintain the propagation of gExEn cells under feeder-free conditions (data not shown). In contrast, as suggested for the possible involvement of the LIF signal for XEN cell maintenance [12], the addition of LIF in the culture of gExEn cells enhanced their propagation (data not shown). The relationship between the activities of GATA factors and the soluble factor(s) in MEF-CM will be tested using the in vitro model system with gExEn cells and XEN cells.

The role of MEF-derived soluble factor(s) might not be restricted in the transcriptional activation of endogenous Gata6 and Gata4. It has been reported the post-translational modification of GATA4 is important to acquire full transcriptional activity [35]. According to this report, acetylation of GATA4, which might be mediated by p300, increases its DNA-binding, resulting enhancement of its transcriptional activity. It is also possible that this pathway is regulated by MEF-derived factor(s). Efficient maintenance of ExEn cells without MEF by activation of GATA4GR or GATA6GR might reflect their ability to compensate for both signal dependencies on transcription and post-translational modification by an unexpected effect of the fusion to the GR ligand binding domain.

gExEn cells express many ExEn marker genes, including those specific for VE and PE. gExEn cells have the potential to contribute to PE in vivo in chimeras, as do XEN cells, indicating that activation of Gata4 or Gata6 is sufficient to induce proper differentiation of XEN-like cells from ES cells. However, as is the case for XEN cells, gExEn cells exhibit a strong bias to contribute to PE in chimeric 
embryos. Indeed, Gata6 is required for VE formation; Gata6-null ES cells fail to differentiate into VE on the surface of embryoid body $[4,6,7]$. During Dex withdrawalinduced differentiation of early passage gExEn cells in vitro, their morphology became flattened with ruffled membranes, reminiscent of VE following upregulation of VE markers, as found in XEN cells. Blastocyst injection showed, however, that PrE and nascent VE cells directly isolated from embryos contributed mostly to PE, indicating that PrE or VE dissected from ICM or epiblast tends to become PE [23]. Interestingly, Casanova and Grabel [36] reported that VE-like cells derived from the embryoid bodies of F9 embryonal carcinoma cells maintain the VE phenotype on the surface of EB or gelatin-coated dextran beads but lose it rapidly under monolayer conditions, with repression of the VE marker $A f p$ and activation of the PE marker Plat. Therefore, the bias of gExEn and XEN cells to the PE phenotype may be due to 2-dimensional culture conditions, which are not permissive for maintenance of the VE phenotype.

As previously shown, we found that artificially-expressed Gata4 or Gata6 activated both endogenous Gata4 and Gata6 [10] to maintain the propagation of gExEn cells. After 5 passages, however, gExEn cells gradually acquire the ability to propagate without activation of exogenous Gata factors. Such weaning from exogenous GATA activity may be achieved by locking the auto-regulatory positive feedback loop between endogenous Gata4 and Gata6. This may mimic the situation in vivo, where transient exogenous signals activating the expression of Gata factors are required to generate the mature ExEn cell population that propagates continuously as it expands along the yolk sac. It is also possible to regard this phenomenon as an artificial condition generated by continuous activation of GATA factors at high levels. In any case, since the embryoderived XEN cells never proliferate without MEF, the balance of the transcription factors in these cell lines should be different, and a global comparison of their transcriptomes will provide a cue to solve the structure of the transcription factor network including Gata4 and Gata6 in ExEn cells.

\section{Conclusion}

We have succeeded in the establishment of ExEn cell lines, that have the same character of XEN cells derived from embryos, from ES cells by the constitutive activation of ExEn specific transcription factor, GATA4 and GATA6.

Establishment of gExEn cells, as with TS cells [37], from ES cells confirmed that the two differentiation events in mouse pre-implantation development could be mimicked by the in vitro activation of lineage-specific transcription factors. This model can be regarded as a powerful tool for investigating the transcriptional network transition from pluripotent stem cells to lineage-restricted cells.

\section{Methods \\ Plasmid construction}

DNA manipulations were performed by standard procedures [38]. Full details of plasmid constructions are available on request.

To generate Gata6-GR or Gata4-GR chimeric genes (designated G6GR and G4GR, respectively), the cDNA fragment encoding the ligand-binding domain (LBD) of the human glucocorticoid receptor (GR) was amplified by PCR, using the oligonucleotide primers, 5'-ACCATGGAAAATCCTGGTAACAAAACA-3' and 5'-ATGCGGCCGCTCACTTTTGATGAAACAGAAG-3', which contained NcoI and NotI restriction sites (underlined), respectively. The fragment was ligated into the NcoI and NotI sites of $p C A G-c H A-I P$ (a derivative of $p C A G-I P$ : [39]), resulting in the generation of pCAG-chGR-IP. Full-length mouse Gata4 and Gata6 cDNAs were PCR amplified from pCAG-Gata4-IP and pCAG-Gata6-IP, respectively, using the oligonucleotide primers, 5'-CCTCGAGCTTGGGGCGATGTACCAA-3' and 5'-AATCATGACCGCGGTGATTATCTCCCCATG-3' for Gata4, and 5'-TTCTCGAGCAGCCGGAGGAAATGTACC$3^{\prime}$ and 5'-AATCATGAGGGCCAGAGCACACCAAGAATC3' for Gata6, each set of which contained XhoI and BspHI restriction sites (underlined) [10], and inserted into the Xhol and NcoI sites of $p C A G-c h G R-I P$, generating $p C A G$ G6GR-IP and $p C A G-G 4 G R-I P$, respectively.

To visualize nuclear translocation of the chimeric GR protein, $p C A G-E G F P-G 6 G R$ was constructed by in-frame insertion of EGFP upstream of G6GR.

\section{Gene silencing}

We used pSilencer $3.1 \mathrm{H} 1$ puro vector (pSil-H1puro; Ambion) for gene silencing. Specific hairpin-forming inserts containing the 19-mer siRNA target sequence of Gata6, 5'-TGCGTTGCAGCAATCAGTG-3' (N19) [40], a linker sequence (5'-TAGTGAAGCCACAGATGTA-3'), and six thymidines as a termination signal were generated using a pair of nucleotides, 5'-GGATCCTGAGCGA(senseN19)-(linker)-(antisenseN19)-GTGCCTATTTTTTGGAAA-3', which included a BamHI site (underlined), and 5'-AAGCTTTTCCAAAAAATAGGCAC-(senseN19)(TACATCTGTGGCTTCACTA-linker)-(antisenseN19)-

TCGCTCAG-3', which included a HindIII site (underlined). After annealing these oligonucleotides, the resulting double-stranded fragments were ligated into the BamHI and HindIII sites of pSil-H1puro, resulting in the generation of $p S i l-s h G 6$ and the hairpin-forming inserts were sequenced using an $\mathrm{ABI} 3130 \mathrm{xl}$ genetic analyzer. 
For the transfection of $p S i l-s h G 6$, we established another 5G6GR cell lines carrying pCAG-Gata6GR-IRES-HisD expression vector, designated for 1D3. 1D3 cells can differentiate to ExEn and propagate in the presence of Dex condition as same as 5G6GR cells.

\section{Cell culture and transfection}

All ES cells were cultured on gelatin-coated dishes in the absence of feeder cells in Glasgow minimal essential medium (GMEM; Sigma) supplemented with 10\% fetal calf serum (FCS), $1 \mathrm{mM}$ sodium pyruvate (Invitrogen), 10${ }^{4}$ M 2-mercaptoethanol, $1 \times$ non-essential amino acids (Invitrogen) and $1000 \mathrm{U} / \mathrm{ml}$ of LIF.

Transfection of the expression vectors into ES cells was performed as described using Lipofectoamine 2000 (Invitrogen) [10]. 5G6GR and 5G4GR ES cells were generated by random integration of the linearized Gata6-GR and Gata4-GR expression vectors, respectively, into E14tg2aderived EB5 ES cells, in which one endogenous Oct3/4 allele is disrupted by a blasticidin resistance gene [41].

For Gata-GR activation, $100 \mathrm{mM}$ dexamethasone (Dex: Sigma) was added to the culture of 5G6GR or 5G4GR ES cells, with the resulting ExEn cells designated g6ExEn and g4ExEn cells, respectively. These gExEn cells were cultured using the same conditions as ES cells, except for withdrawal of LIF. The gExEn cells, which can propagate without Dex, were transiently transfected with gene silencing vectors using the same method as for ES cells.

\section{Luciferase reporter assay}

For the construction of $p \mathrm{Fgf3}-\mathrm{luc}$ vector, fragment of DNA encompassing $1.7 \mathrm{~kb}$ of sequence immediately $5^{\prime}$ of the Fgf3 coding region containing GATA binding site [42] was PCR amplified from BAC containing 5' sequence of $F g f 3$ genomic region using the oligonucleotide primers, 5'AAAGGATTCAGATGCCCTCTGGAT-3', which included a BamHI site (underlined), and 5'-TTTGCCGGCTCGACTGTGGCTA-3', which included a NaeI site (underlined), and inserted into the BglII and HIndIII (Blunted) sites of pGL3 (Promega).

For transfection of reporter plasmids, $1 \times 10^{4}$ cells were seeded in each well of a 96-well plate and incubated with $0.33 \mu \mathrm{g}$ reporter plasmid and $0.33 \mathrm{ng}$ of the internal control plasmid pRL-CMV, together with Lipofectoamine 2000 (Invitrogen), following the manufacturer's protocol. Luciferase assays were performed 24 hours later using a Dual-luciferase assay kit (Promega).

\section{Derivation and culture of XEN cells}

Following overnight culture of $3.5 \mathrm{dpc}$ C57Bl/6J blastocysts in KSOM (Specialty Media), the blastocysts were incubated at $37^{\circ} \mathrm{C}$ for $5 \mathrm{~min}$ with $0.5 \% \mathrm{~S}$. griseus protease
(Pronase; Sigma) to remove the zona pellucida, plated on 48-well plates coated with mouse embryonic feeder cells, and cultured in RPMI1640 (Gibco) containing rhFGF4 (25 ng/ml, Wako), following the conditions as described by Kunath et al. [12]. After 7 days, XEN-like cells were passaged 1:1 onto new MEF in 4-well plates; after another 7 days, two lines of XEN cells (XEN2 and XEN9) were passaged in FGF4-free media.

\section{Production of chimeric embryos}

To visualize the in vivo contribution of gExEn cells, 5G6GR-GFP and 5G4GR-GFP ES cells were established by introducing constitutive EGFP expression vector ( $p C A G$ EGFP-IZ) into 5G6GR and 5G4GR ES cells, respectively. To obtain chimeric embryos, ES and g6ExEn-GFP or g4ExEn-GFP cells were injected into C57Bl/6J blastocysts (2-3 cells per blastocyst), followed by transfer into the uteri of pseudopregnant ICR mice.

Embryos were dissected at $8.5 d p c$ and fluorescent signals were detected using an Olympus SZX12 fluorescent dissecting microscope and captured with an Olympus DP70 cooled color digital (CCD) camera.

\section{RNA preparation and real-time PCR}

Total RNA was prepared using TRIzol reagent (Invitrogen) according to the manufacturer's instructions. First strand cDNA was synthesized from $1 \mu \mathrm{g}$ of total RNA in $40 \mu \mathrm{l}$ containing oligo-dT primers using a ReverTra Ace firststrand synthesis kit (Toyobo). Real-time PCR was performed with the ExTaq cyber green supermix (Takara) using an iCycler System (Bio-Rad). The amount of target RNA was determined from the appropriate standard curve, and was normalized relative to the amount of Gapdh mRNA. Sequences of primers for QPCR are listed on Table 3. Gata6 or Gata4 primer pairs were designed to amplify 3' untranslated regions, and thus to detect only endogenous transcripts.

\section{Immunostaining}

Cells were fixed with $10 \%$ formaldehyde for $5 \mathrm{~min}$ at room temperature, washed with PBS containing $0.5 \%$ Triton $\mathrm{X}-100$ for $10 \mathrm{~min}$ at room temperature, and incubated with rabbit anti-Gata6\#1 antibody that we raised by immunization with GST-GATA6 fusion protein followed by affinity purification and anti-Disabled-2 (p96) monoclonal Ab (610464, BD transduction). The cells were incubated with anti-rabbit or anti-mouse IgG secondary antibodies conjugated with Alexa Fluor 594 or 488 (Molecular Probes), respectively. Fluorescent images were captured with an IX51 microscope (Olympus; Tokyo, Japan) and DP70 Digital camera (Olympus). 
Table 3: Primer Sequences for Q-PCR

\begin{tabular}{|c|c|c|c|c|c|}
\hline Gene & Primer & Sequence & Gene & Primer & Sequence \\
\hline \multirow[t]{2}{*}{ Gata6 } & $\mathrm{F}$ & GAGCTGGTGCTACCAAGAGG & Plat & $\mathrm{F}$ & GATGACAGGGAGATGCCAAC \\
\hline & $\mathrm{R}$ & TGCAAAAGCCCATCTCTTCT & & $\mathrm{R}$ & CTTGTCCCCAGTGCAAACTT \\
\hline \multirow[t]{2}{*}{ Gata4 } & $\mathrm{F}$ & СССТТСССТСТТСАААТТСС & $m S n a$ & $\mathrm{~F}$ & GCTGTGTTGGAAACGGAGTT \\
\hline & $\mathrm{R}$ & CTTTTCCAGAGCTCCACCTG & & $\mathrm{R}$ & CATGTGGGTTCTGACTGGTG \\
\hline \multirow[t]{2}{*}{ Sox 7} & $\mathrm{~F}$ & GCTCCTGCTTTTGGTGTAGC & SPARC & $\mathrm{F}$ & GTTCCTGCTTGGCTCTCTTG \\
\hline & $\mathrm{R}$ & GTCCTTGGGCAGTCATTCAT & & $\mathrm{R}$ & CCTTGAGGGAGGTAGGGAAG \\
\hline \multirow[t]{2}{*}{ Sox 17} & $\mathrm{~F}$ & GAGGGCCAGAAGCAGTGTTA & Follistatin & $\mathrm{F}$ & ACCTGAGAAAGGCCACCTG \\
\hline & $\mathrm{R}$ & AGTGATTGTGGGGAGCAAGT & & $\mathrm{R}$ & AGCTTCCTTCATGGCACACT \\
\hline \multirow[t]{2}{*}{ Dab2 } & $\mathrm{F}$ & TCTCAGCCTGCATCTTCTGA & Pthrl & $\mathrm{F}$ & AGGACGACGGCTTCCTTAAT \\
\hline & $\mathrm{R}$ & GAGCGAGGACAGAGGTCAAC & & $\mathrm{R}$ & TTGTCTTCCTGGTCCAGTCC \\
\hline \multirow[t]{2}{*}{ AFP } & $\mathrm{F}$ & TCCAGAAGGAAGAGTGGACAA & Oct3/4 & $\mathrm{F}$ & CACGAGTGGAAAGCAACTCA \\
\hline & $\mathrm{R}$ & GCAGACTAGGAGAAGAGAAATAGTTGA & & $\mathrm{R}$ & AGATGGTGGTCTGGCTGAAC \\
\hline \multirow[t]{2}{*}{ ApoE } & $\mathrm{F}$ & GGTTCGAGCCAATAGTGGAA & Nanog & $\mathrm{F}$ & CACCCACCCATGCTAGTCTT \\
\hline & $\mathrm{R}$ & TATTAAGCAAGGGCCACCAG & & $\mathrm{R}$ & ACССTCAAACTCСTGGTCCT \\
\hline \multirow[t]{2}{*}{ RBP } & $\mathrm{F}$ & GAACTTCGACAAGGCTCGTTTCTCGG & RexI & $\mathrm{F}$ & GAGTTCGTCCATCTAAAAAGGGAGG \\
\hline & $\mathrm{R}$ & ATCCAGTGGTCATCGTTTCCTCGCT & & $\mathrm{R}$ & TCTTAGCTGCTTCCTTGAACAATGCC \\
\hline \multirow[t]{2}{*}{ Ihh } & $\mathrm{F}$ & GGCCTGGGATTGTGACTTTA & $C d \times 2$ & $\mathrm{~F}$ & AGGCTGAGCCATGAGGAGTA \\
\hline & $\mathrm{R}$ & CTGCAGGGAAGGTCATGTTT & & $\mathrm{R}$ & CGAGGTCCATAATTCCACTCA \\
\hline \multirow[t]{2}{*}{$H n f 3 b$} & $\mathrm{~F}$ & CCCTGCTAGCTCTGGTCACT & HandI & $\mathrm{F}$ & ССССТCTTCСGTCСTCTTAC \\
\hline & $\mathrm{R}$ & ACAGATCACTGTGGCCCATC & & $\mathrm{R}$ & CTGCGAGTGGTCACACTGAT \\
\hline \multirow[t]{2}{*}{ Msg2 } & $\mathrm{F}$ & ATGCCAACCAGGAGATGAAC & Psxl & $\mathrm{F}$ & GAATTGGTTTCGGATGAGGA \\
\hline & $\mathrm{R}$ & AGGATGCAGGTTGAAGGATG & & $\mathrm{R}$ & GTGGCTCAGAAGAAGCCATC \\
\hline \multirow[t]{2}{*}{ Ttr } & $\mathrm{F}$ & CTCACCACAGATGAGAAG & Gapdh & $\mathrm{F}$ & ACCACAGTCCATGCCATCAC \\
\hline & $\mathrm{R}$ & GGCTGAGTCTCTCAATTC & & $\mathrm{R}$ & TCCACCACCCTGTTGCTGTA \\
\hline
\end{tabular}

$F$, forward; R, reverse

\section{Competing interests}

The author(s) declare that they have no competing interests.

\section{Authors' contributions}

DS carried out almost all of the experiments, helped conceive the study and drafted the manuscript. MS performed FACS analysis. HN conceived the study, reviewed and analyzed all data and drafted the manuscript. All authors read and approved the final manuscript.

\section{Additional material}

\section{Additional file 1}

Activation of GATA-dependent reporters by GATA6 or GATA4 in ES cells. Activities of pGata6-luc and pFgf3-tk-luc in ES cells carrying various inducible GATA expression units with or without induction.

Click here for file

[http://www.biomedcentral.com/content/supplementary/1471213X-7-80-S1.doc]

\section{Acknowledgements}

We thank all members of our laboratory for helpful discussions. This research was supported by a RIKEN grant and grants for the 2 I $^{\text {st }}$ century COE program, "Center of Excellence for Signal Transduction Disease: Diabetes Mellitus as Model", from the Ministry of Education, Culture, Sports, Science and Technology of Japan and the Leading Project (to H.N.). H.N. also received funding from the CREST program of the Japan Science and Technology Agency on the research subject: The High Throughput Creation of Disease Model Cells and the Analysis of Their Function.

\section{References}

I. Enders AC, Schlafke S: Comparative aspects of blastocystendometrial interactions at implantation. Ciba Found Symp 1978:3-32.

2. Hogan BL, Cooper AR, Kurkinen $M$ : Incorporation into Reichert's membrane of laminin-like extracellular proteins synthesized by parietal endoderm cells of the mouse embryo. Dev Biol 1980, 80:289-300.

3. Morrisey EE, Ip HS, Lu MM, Parmacek MS: GATA-6: a zinc finger transcription factor that is expressed in multiple cell lineages derived from lateral mesoderm. Dev Biol 1996, 177:309-322.

4. Koutsourakis M, Langeveld A, Patient R, Beddington R, Grosveld F: The transcription factor GATA6 is essential for early extraembryonic development. Development 1999, I 26(9):723-732.

5. Chazaud C, Yamanaka Y, Pawson T, Rossant J: Early lineage segregation between epiblast and primitive endoderm in mouse blastocysts through the Grb2-MAPK pathway. Dev Cell 2006, 10:615-624.

6. Morrisey EE, Tang Z, Sigrist K, Lu MM, jiang F, Ip HS, Parmacek MS: GATA6 regulates HNF4 and is required for differentiation of visceral endoderm in the mouse embryo. Genes Dev 1998, I 2:3579-3590.

7. Capo-Chichi CD, Rula ME, Smedberg JL, Vanderveer L, Parmacek MS, Morrisey EE, Godwin AK, Xu XX: Perception of differentiation cues by GATA factors in primitive endoderm lineage determination of mouse embryonic stem cells. Dev Biol 2005, 286:574-586.

8. Smith AG, Heath JK, Donaldson DD, Wong GG, Moreau J, Stahl M, Rogers D: Inhibition of pluripotential embryonic stem cell differentiation by purified polypeptides. Nature 1988, 336:688-690.

9. Niwa H, Miyazaki J, Smith AG: Quantitative expression of Oct-3/ 4 defines differentiation, dedifferentiation or self-renewal of ES cells. Nat Genet 2000, 24:372-376. 
10. Fujikura ], Yamato E, Yonemura S, Hosoda K, Masui S, Nakao K, Miyazaki Ji J, Niwa H: Differentiation of embryonic stem cells is induced by GATA factors. Genes Dev 2002, 16:784-789.

II. Beddington RS, Robertson EJ: An assessment of the developmental potential of embryonic stem cells in the midgestation mouse embryo. Development 1989, 105:733-737.

12. Kunath T, Arnaud D, Uy GD, Okamoto I, Chureau C, Yamanaka Y, Heard E, Gardner RL, Avner P, Rossant J: Imprinted X-inactivation in extra-embryonic endoderm cell lines from mouse blastocysts. Development 2005, I32:|649-|66I.

13. Masui S, Shimosato D, Toyooka Y, Yagi R, Takahashi K, Niwa H: An efficient system to establish multiple embryonic stem cell lines carrying an inducible expression unit. Nucleic Acids Res 2005, 33:e43.

14. Kobayashi M, Nishikawa K, Yamamoto M: Hematopoietic regulatory domain of gata I gene is positively regulated by GATA I protein in zebrafish embryos. Development 200I, I 28:234I-2350.

15. Murakami A, Thurlow J, Dickson C: Retinoic acid-regulated expression of fibroblast growth factor 3 requires the interaction between a novel transcription factor and GATA-4. J Biol Chem 1999, 274:I7242-I7248.

16. Arceci RJ, King AA, Simon MC, Orkin SH, Wilson DB. Mouse GATA-4: a retinoic acid-inducible GATA-binding transcription factor expressed in endodermally derived tissues and heart. Mol Cell Biol I993, I 3:2235-2246.

17. Kanai-Azuma M, Kanai Y, Gad JM, Tajima Y, Taya C, Kurohmaru M, Sanai Y, Yonekawa H, Yazaki K, Tam PP, Hayashi Y: Depletion of definitive gut endoderm in Sox I7-null mutant mice. Development 2002, I 29:2367-2379.

18. Yang DH, Smith ER, Roland IH, Sheng Z, He J, Martin WD, Hamilton TC, Lambeth JD, Xu XX: Disabled-2 is essential for endodermal cell positioning and structure formation during mouse embryogenesis. Dev Biol 2002, 25 I:27-44.

19. Marotti KR, Belin D, Strickland S: The production of distinct forms of plasminogen activator by mouse embryonic cells. Dev Biol I 982, 90:154-159.

20. Veltmaat JM, Orelio CC, Ward-Van Oostwaard D, Van Rooijen MA, Mummery CL, Defize LH: Snail is an immediate early target gene of parathyroid hormone related peptide signaling in parietal endoderm formation. Int J Dev Biol 2000, 44:297-307.

21. Mason IJ, Taylor A, Williams JG, Sage H, Hogan BL: Evidence from molecular cloning that SPARC, a major product of mouse embryo parietal endoderm, is related to an endothelial cell 'culture shock' glycoprotein of Mr 43,000. Embo J 1986 , 5: $1465-1472$

22. Feijen A, Goumans MJ, van den Eijnden-van Raaij AJ: Expression of activin subunits, activin receptors and follistatin in postimplantation mouse embryos suggests specific developmental functions for different activins. Development 1994 120:362I-3637.

23. Verheijen $M H$, Karperien $M$, Chung $U$, van Wijuen $M$, Heystek $H$, Hendriks JA, Veltmaat JM, Lanske B, Li E, Lowik CW, de Laat SW, Kronenberg HM, Defize LH: Parathyroid hormone-related peptide (PTHrP) induces parietal endoderm formation exclusively via the type I PTH/PTHrP receptor. Mech Dev 1999 , 8I:|5|-|6I.

24. Dziadek M, Adamson E: Localization and synthesis of alphafoetoprotein in post-implantation mouse embryos. J Embryol Exp Morphol 1978, 43:289-3।3.

25. Dufort D, Schwartz L, Harpal K, Rossant J: The transcription factor HNF3beta is required in visceral endoderm for normal primitive streak morphogenesis. Development 1998 , 125:3015-3025.

26. Maye P, Becker S, Kasameyer E, Byrd N, Grabel L: Indian hedgehog signaling in extraembryonic endoderm and ectoderm differentiation in ES embryoid bodies. Mech Dev 2000, 94:1 I7-132.

27. Abe K, Niwa H, Iwase K, Takiguchi M, Mori M, Abe SI, Yamamura KI: Endoderm-specific gene expression in embryonic stem cells differentiated to embryoid bodies. Exp Cell Res 1996, 229:27-34.

28. Soprano DR, Soprano KJ, Wyatt ML, Goodman DS: Induction of the expression of retinol-binding protein and transthyretin in F9 embryonal carcinoma cells differentiated to embryoid bodies. J Biol Chem 1988, 263:17897-17900.

29. Basheeruddin K, Stein P, Strickland S, Williams DL: Expression of the murine apolipoprotein $E$ gene is coupled to the differen- tiated state of F9 embryonal carcinoma cells. Proc Natl Acad Sci U S A 1987, 84:709-7I3.

30. Dunwoodie SL, Rodriguez TA, Beddington RS: Msg I and MrgI, founding members of a gene family, show distinct patterns of gene expression during mouse embryogenesis. Mech Dev 1998, 72:27-40.

31. Niwa $\mathrm{H}$ : How is pluripotency determined and maintained? Development 2007, I 34:635-646.

32. Wewer U: Characterization of a rat yolk sac carcinoma cell line. Dev Biol 1982, 93:416-42I.

33. Notarianni E, Flechon J: Parietal endoderm cell line from a rat blastocyst. Placenta 200I, 22: I I I-I23.

34. Fowler KJ, Mitrangas K, Dziadek M: In vitro production of Reichert's membrane by mouse embryo-derived parietal endoderm cell lines. Exp Cell Res 1990, I 9 I: 194-203.

35. Kawamura T, Ono K, Morimoto T, Wada H, Hirai M, Hidaka K, Morisaki T, Heike T, Nakahata T, Kita T, Hasegawa K: Acetylation of GATA-4 is involved in the differentiation of embryonic stem cells into cardiac myocytes. J Biol Chem 2005, 280: I9682-19688.

36. Casanova JE, Grabel LB: The role of cell interactions in the differentiation of teratocarcinoma-derived parietal and visceral endoderm. Dev Biol 1988, I 29:124-139.

37. Niwa H, Toyooka Y, Shimosato D, Strumpf D, Takahashi K, Yagi R, Rossant J: Interaction between Oct3/4 and Cdx2 determines trophectoderm differentiation. Cell 2005, I 23:917-929.

38. Sambrook J E.F. Fritsch, and T. Maniatis.: Molecular cloning: A laboratory manual. Cold Spring Harbor, NY., Cold Spring Harbor Laboratory Press.; 1989.

39. Niwa H, Masui S, Chambers I, Smith AG, Miyazaki J: Phenotypic complementation establishes requirements for specific $\mathrm{POU}$ domain and generic transactivation function of Oct-3/4 in embryonic stem cells. Mol Cell Biol 2002, 22:I526-I536.

40. Futaki S, Hayashi Y, Emoto T, Weber CN, Sekiguchi K: Sox7 plays crucial roles in parietal endoderm differentiation in $\mathbf{F 9}$ embryonal carcinoma cells through regulating Gata-4 and Gata-6 expression. Mol Cell Biol 2004, 24: I0492-10503.

4I. Kawasaki H, Mizuseki K, Nishikawa S, Kaneko S, Kuwana Y, Nakanishi $S$, Nishikawa SI, Sasai Y: Induction of midbrain dopaminergic neurons from ES cells by stromal cell-derived inducing activity. Neuron 2000, 28:31-40.

42. Murakami A, Grinberg D, Thurlow J, Dickson C: Identification of positive and negative regulatory elements involved in the retinoic acid/cAMP induction of Fgf-3 transcription in F9 cells. Nucleic Acids Res 1993, 2 I:5351-5359.

Publish with BioMed Central and every scientist can read your work free of charge

"BioMed Central will be the most significant development for disseminating the results of biomedical research in our lifetime. "

Sir Paul Nurse, Cancer Research UK

Your research papers will be:

- available free of charge to the entire biomedical community

- peer reviewed and published immediately upon acceptance

- cited in PubMed and archived on PubMed Centra

- yours - you keep the copyright 